\title{
An Infrequent Type of Stroke with an Unusual Cause and Successful Therapy: Basilar Artery Occlusion Caused by a Cardiac Papillary Fibroelastoma Recanalized 12 Hours after Onset
}

\author{
Josip Ljevak $^{1}$, Antonija Mišmaš ${ }^{1}$, Antonela Bazina ${ }^{1}$, Vesna Matijević ${ }^{1}$, Domagoj Alvir ${ }^{1}$, \\ Svjetlana Šupe ${ }^{1}$, Snježana Juren Meaški ${ }^{1}$, David Ozretić ${ }^{2}$, \\ Zdravka Poljaković $^{1,3}$ and Mario Habek ${ }^{1,3}$
}

\begin{abstract}
We herein report the case of a 32-year-old woman with sudden onset ataxia, limb dysmetria and somnolence. Emergency radiological findings showed bilateral cerebellar and thalamic infarctions as a result of a basilar artery occlusion. The patient was treated with intra-arterial (IA) and mechanical thrombolysis 12 hours after symptom onset and showed an excellent recovery. A diagnostic workup revealed a tumor mass on the mitral valve that was surgically removed, while a histological analysis confirmed a diagnosis of cardiac papillary fibroelastoma.
\end{abstract}

Key words: basilar artery occlusion, intra-arterial thrombolysis, mechanical thrombolysis, cardiac papillary fibroelastoma

(Intern Med 52: 277-279, 2013)

(DOI: 10.2169/internalmedicine.52.8739)

\section{Introduction}

Acute basilar artery occlusion (BAO) is, in the majority of patients, a devastating neurological condition associated with a high risk of long-term disability and death. Despite this well-known fact and the availability of several different treatment options, there are still no definitive treatment guidelines. We herein present the case of a young patient diagnosed with an acute stroke caused by BAO who was successfully treated with a combination of intra-arterial and mechanical thrombolysis performed with a substantial time delay. A further workup revealed that this incident was the first presentation of a rare benign cardiac tumor.

\section{Case Report}

A 32-year-old woman was brought to our emergency de- partment following the sudden onset of disturbed consciousness, vertigo with instability, nausea and vomiting. An initial clinical examination revealed somnolence, dysarthria, horizontal left-beating nystagmus, a positive Romberg sign and bilateral limb ataxia. Urgent brain magnetic resonance imaging (MRI) showed multiple ischemic lesions in both cerebellar hemispheres and both thalami without signs of intracranial hemorrhage, while magnetic resonance angiography (MRA) demonstrated occlusion of the distal third of the basilar artery (Fig. 1). Transthoracic echocardiography (TTE) performed in the Emergency Department (ED) showed only minimal mitral insufficiency with no visible tumor masses. Immediate cerebral digital subtraction angiography (DSA) confirmed the presence of an acute BAO distal to the origin of the anterior inferior cerebellar arteries, with sustained circulation through the posterior cerebral arteries without any signs of vertebral artery dissection (Fig. 2). A microcatheter (Headway-17, Microvention) was then navi-

${ }^{1}$ Department of Neurology, University Hospital Center Zagreb, Croatia, ${ }^{2}$ Department of Radiology, University Hospital Center Zagreb, Croatia and ${ }^{3}$ School of Medicine, University of Zagreb, Croatia

Received for publication August 4, 2012; Accepted for publication October 3, 2012

Correspondence to Dr. Mario Habek, mhabek@mef.hr 


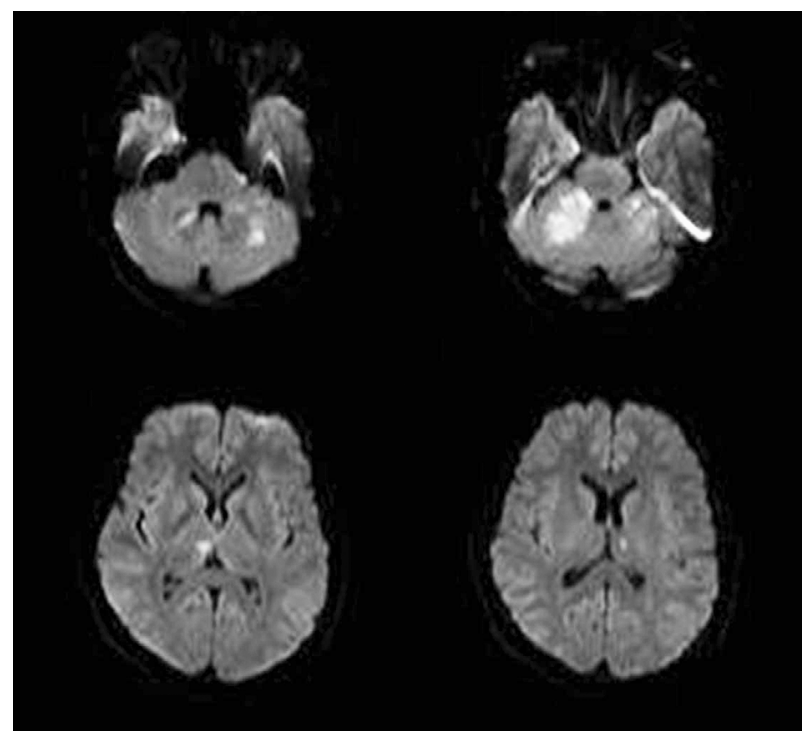

Figure 1. Prethrombolysis dwi $\left(b=1,000 \mathrm{~mm}^{2} / \mathrm{s}\right)$ showing bilateral lesions in the cerebellum and thalami.

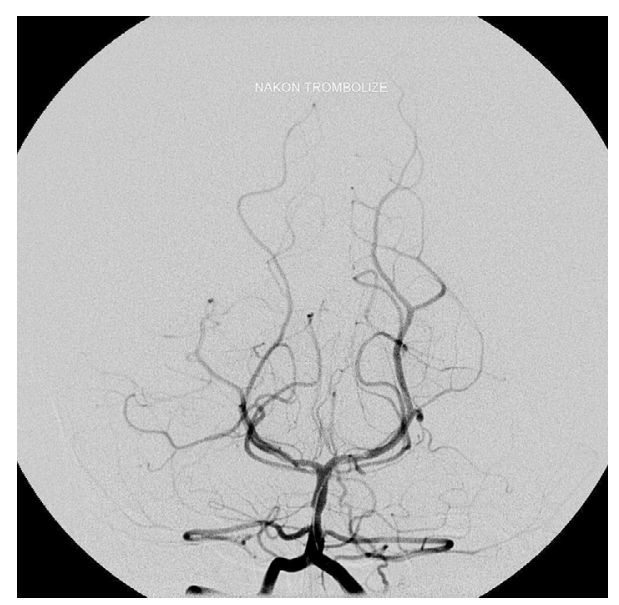

Figure 3. Vertebral artery recanalization after IA thrombolysis.

gated through the thrombus, and the patency of both posterior cerebral arteries was assessed. The microcatheter was then withdrawn, and an infusion of r-tPA in front and inside of the thrombus was started. After the administration of 50 $\mathrm{mg}$ of r-tPA, control angiography showed recanalization of the basilar artery with residual stenosis (up to 50\%) at the site of the thrombus and persistent occlusion of the left posterior cerebral artery (PCA). The residual clot in the left PCA was then mechanically disrupted using microwire (Agility-14, Codman) manipulation. Following this maneuver, appropriate distal filling was achieved (Fig. 3). Additionally, $4 \mathrm{mg}$ of r-tPA were administered at the site of the basilar artery stenosis without evident effects. The procedure was terminated when a full dose of r-tPA was reached. Further mechanical fragmentation was aborted due to the risk of perforator occlusion. Follow-up brain MRI performed after nine days showed bilateral ischemic lesions in the pons, cerebellum and thalami, while cerebral MRA continued to show basilar artery stenosis. At that time, the patient exhib-

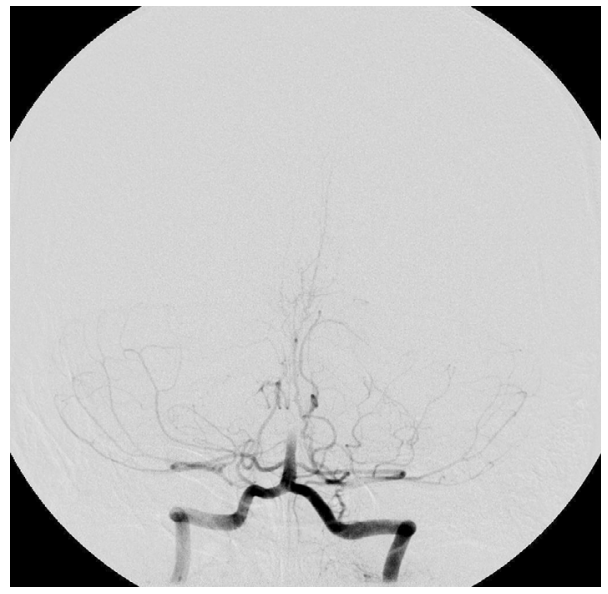

Figure 2. Distal vertebral artery occlusion as seen on initial DSA.

ited only mild right hand ataxia and mild dysarthria. The laboratory findings, including extensive hematology, immunology and coagulation tests, showed only mild anemia and a urinary tract infection, which was treated with antibiotics, and were otherwise unremarkable. The carotid and vertebral artery ultrasonography findings were normal, as were the results of a 24-hour electrocardiogram. A transesophageal echocardiogram (TEE) showed a small tumor mass adherent to the anterior leaflet of the mitral valve. After achieving an excellent neurological recovery, the patient underwent surgical resection of the heart tumor. A pathologic analysis confirmed the diagnosis of papillary fibroelastoma. On a follow-up examination conducted after six months, the patient was found to have no neurologic deficits and the cerebral MRA findings were completely normal.

\section{Discussion}

We herein present the case of a 32-year-old woman with a basilar artery occlusion that was successfully treated with combined IA and mechanical thrombolysis 12 hours after the onset of the initial symptoms. A diagnostic workup revealed a rare benign cardiac tumor as the source of the cerebral embolus. Compared to anterior circulation strokes, basilar artery occlusions occur less frequently; however, without recanalization, BAOs are associated with an extremely high mortality rate. Current treatment guidelines restrict the use of IA thrombolysis to six hours from the onset of symptoms caused by occlusion of the MCA, with a number of reported cases of successful treatment administered over prolonged time periods after the initial onset of symptoms $(1,2)$. Randomized controlled data on the treatment of acute BAO are lacking and focus primarily on IV or IA thrombolysis. Available studies on mechanical thrombolysis show different results, with some suggesting the superiority of mechanical thrombolysis as a separate treatment (3) and others showing no statistically significant superiority of any treatment strategy (4). Considering the poor prognoses and outcomes of patients with basilar artery occlusions, there is no strict time 
restriction for the use of local thrombolytic therapy to treat BAOs.

In this particular case, the patient presented to the ED approximately nine hours after the onset of symptoms because her initial symptoms (nausea and vomiting) were regarded as symptoms of an infectious gastric disturbance, thus being ineligible for IV thrombolysis. Given the severity of her neurological deficits, the radiological findings of multiple infarctions and BAO, local thrombolysis was performed despite the fact that 12 hours had passed after the onset of symptoms. The patient's clinical recovery was excellent.

The source of the cerebral embolus, cardiac papillary fibroelastoma (PFE), is the third most common type of heart tumor and occurs primarily in persons over the age of 50 with no sex preferences. PFEs are typically asymptomatic, although they remain a possible source of cerebral emboli, either from the tumor itself or from fibrin thrombi attached to its surface $(5,6)$. In this case, r-TPA therapy achieved partial recanalization of the basilar artery; therefore, the mechanical clot disruption that followed was necessary to achieve additional thrombolytic effects. Thrombectomy was not performed; hence, further pathohistological analyses of the embolus were not possible. Based on the limited effects of the locally applied full dose of r-TPA and the success of mechanical thrombolysis, we can speculate that the embolus may have been composed of both fibrin and tumor tissue, or less probably, fibrin only.

There are no randomized controlled studies on the application of thrombolytic agents in patients with cardiac tumorinduced strokes. To the best of our knowledge, the literature contains only two reports of thrombolytic agents being successfully used to treat PFE-associated strokes: one case reported successful intravenous (IV) thrombolysis (6) and one case reported successful intra-arterial thrombolysis (7). In both cases, the strokes were in the anterior circulation. In addition, we found only one case involving the use of mechanical thrombolysis in the treatment of a stroke caused by a cardiac myxoma embolus (8).

In conclusion, this is the first case in which the initial and only clinical presentation of a rare cardiac tumor was a severe posterior circulation stroke that was successfully treated with delayed IA thrombolysis combined with mechanical clot disruption. Adding to this rare etiology, we believe that the most aggressive treatment of this form of stroke was jus- tified, despite the risks and substantial time delay.

The authors state that they have no Conflict of Interest (COI).

\section{Authors' contributions}

Study concept and design: Juren, Ozretić, Poljaković, Habek. Acquisition of data: Ljevak, Mišmaš, Bazina, Matijević, Alvir, Juren, Ozretić, Poljaković, Habek. Analysis and interpretation of data: Ljevak, Mišmaš, Bazina, Matijević, Alvir, Juren, Ozretić, Poljaković, Habek. Drafting of the manuscript: Ljevak, Habek. Critical revision of the manuscript for important intellectual content: Ljevak, Mišmaš, Bazina, Matijević, Alvir, Juren, Ozretić, Poljaković, Habek. Administrative, technical, and material support: Ljevak, Mišmaš, Bazina, Matijević, Alvir, Juren, Ozretić, Poljaković, Habek.

\section{References}

1. Natarajan SK, Snyder KV, Siddiqui AH, Ionita CC, Hopkins LN, Levy EI. Safety and effectiveness of endovascular therapy after 8 hours of acute ischemic stroke onset and wake-up strokes. Stroke 40: 3269-3274, 2009.

2. Barnwell SL, Clark WM, Nguyen TT, O'Neill OR, Wynn ML, Coull BM. Safety and efficacy of delayed intraarterial urokinase therapy with mechanical clot disruption for thromboembolic stroke. AJNR Am J Neuroradiol 15: 1817-1822, 1994.

3. Andersson T, Kuntze Söderqvist A, Söderman M, Holmin S, Wahlgren N, Kaijser M. Mechanical thrombectomy as the primary treatment for acute basilar artery occlusion: experience from 5 years of practice. J Neurointerv Surg 2012 (in press).

4. Schonewille WJ, Wijman CA, Michel P, et al; BASICS study group. Treatment and outcomes of acute basilar artery occlusion in the Basilar Artery International Cooperation Study (BASICS): a prospective registry study. Lancet Neurol 8: 724-730, 2009.

5. Gowda RM, Khan IA, Nair CK, Mehta NJ, Vasavada BC, Sacchi TJ. Cardiac papillary fibroelastoma: a comprehensive analysis of 725 cases. Am Heart J 146: 404-410, 2003.

6. Matijević V, Poljaković Z, Ilić I, Cikeš I, Habek M. Cardiac papillary fibroelastoma: source of cerebral embolism treated with intravenous thrombolysis. J Stroke Cerebrovasc Dis 20: 485-487, 2011.

7. Palazzuoli A, Bruni F, Pasqui A, Puccetti L, Bracco S, Auteri A. Cardiac papillary fibroelastoma: an unusual source of cerebral embolism solved with local fibrinolysis. Neurol Sci 22: 469-472, 2002.

8. Garcia-Ptacek S, Matias-Guiu JA, Valencia-Sánchez C, Gil A, Bernal-Becerra I, De Las Heras-Revilla V, Serna-Candel C. Mechanical endovascular treatment of acute stroke due to cardiac myxoma. J Neurointerv Surg 2012 Jul 12 (in press).

(C) 2013 The Japanese Society of Internal Medicine http://www.naika.or.jp/imonline/index.html 